\title{
Ecological Aspects in Control of Small-scale Biomass Fired Boilers
}

\author{
Bohumil Šulc \\ Department of Instrumentation and Control Engineering \\ Mechanical Engineering Faculty of the CTU in Prague \\ Prague, Czech Republic
}

\author{
Cyril Oswald \\ Department of Instrumentation and Control Engineering \\ Mechanical Engineering Faculty of the CTU in Prague \\ Prague, Czech Republic
}

Received: January 15, 2021. Revised: March 8, 2021. Accepted: March 13, 2021. Published: March 23, 2021.

\begin{abstract}
Control of combustion in small-scale boilers has been standing outside interest for a long time. More attention has been paid to design of the boilers than to economic and ecological aspects of their operation. To the question under which operating conditions of the boiler a controller carries out its activities has been paid a limited attention. The quick by developing use of biomass fired boilers, local shortage of quality biomass, legal restriction on emissions and available cheaper instrumentation have caused a turn in replacing older simple control solutions by more sophisticated technology. The final goal is evident: to achieve for small-scale boilers to be able to operate automatically fulfilling ecological limits comparable with those usual in fullscale boilers, even when firing biomass of lower quality and no skilled service can be expected. Some experience obtained from experiments aimed at this goal and improvements carried out on pilot boilers are reported in this paper.
\end{abstract}

Keywords-biomass boilers; control; emmisions; operating condition optimizitation

\section{INTRODUCTION}

Small-scale biomass boilers were traditionally operated uncontrolled or with a very simple control mechanism. The ecology improvement of such uncontrolled boilers in comparison with fossils fuels can be very small if any. Typical issue is improperly controlled air to fuel ratio during transition states (e.g. boiler lighting, burn-out phase, sudden increase of power demand etc.).

Increasing number of small-scale biomass boiler installations is another important circumstance. Biomass resources become limited, especially those of better quality such pellets making possible very simple and effective on-off control of combustion. Due to this fact more sophisticated control has became the important issue even for small-scale boilers. One of the crucial aspects of economy and ecology of biomass combustion is its local source of fuel. Any transportation of fuels spoils the advantages of using biomass as cheaper and more ecological energy source. Local biomass sources have their limited capacity and thus further growth in large combustion system is not expected.

Nowadays, there are not any emission regulations required for operation of small-scale boilers. For example, in the Czech
Republic are valid operation emission limits established for the boilers with power output above $200 \mathrm{~kW}$. Only for new boilers introduced to the market has been adopted standard EN 303-5. However, current development in the national legislation indicates that in a close future will be established emission limits for the small-scale boilers too.

If we compare conditions for performing control in large boilers with those in small-scale boilers, the main differences are represented by the following points:

- faster fluctuation of combustion process due to smaller inertia of the combustion chamber walls,

- higher sensitivity of combustion process to external influences, load changes, etc

- the small- scale boilers are usually used by the users not having sufficient qualification, therefore periodical maintenance is poor, sensors provide uncertain measurements and manual control is unskilled,

- expenditures for automation of a small-scale boiler has to be kept low because they cannot create the bigger part of the price, etc.

From the control viewpoint combustion of biomass fuels is not a simple matter. If it is required boilers capability to combust various kind of biomass in an optimal way, i.e. with the maximum of efficiency and lowest production of harmful emission we have not enough means how to influence the combustion process.

First of all it is transport of fuel where not only the delivered quantity is important for the boiler's power, but also the way how is the motion of transporter carried out because it is usually linked with grating. In quest of producing the minimal achievable gaseous emissions and maintaining the steady fuel combustion, it is necessary to control the air factor (air excess) $\lambda$, on its desired value. The air factor $\lambda$ is expressed by the ratio

$$
\lambda=\frac{Q_{a}}{Q_{a \min }}>1 \quad[-]
$$


where $Q_{a}$ is the flow rate of actual combustion air and $Q_{a m i n}$ is the necessary (stoichiometric) burning air. The topical value of the air factor $\lambda$ in the running combustion process is acquired via oxygen concentration measurement in the flue gases at the end part of the boiler.

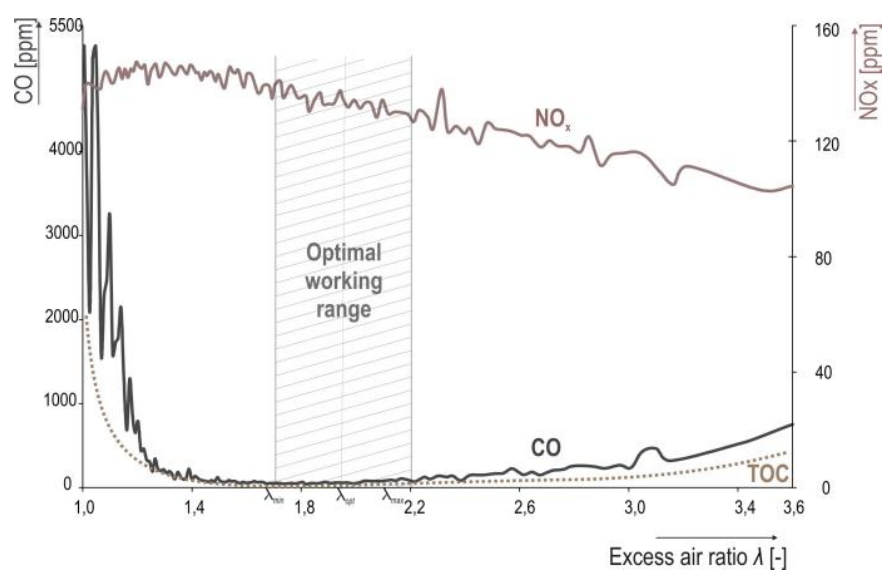

Fig. 1. Dependence of CO, NOx and TOC emissions on the excess air ([1], fig 1)

Fig. 1 demonstrates an important circumstance from the control viewpoint: the optimal working range. If the values of the excess air ratio lie within optimal working range then the burning produces the minimum of carbon monoxide $\mathrm{CO}$ and simultaneously the maximum of efficiency is achieved. On contrary, $\mathrm{NO}_{\mathrm{x}}$ emissions are close to their maximum and therefore higher values of the air excess are accepted as a certain compromise in production of emission. In other words, if we are able to design control algorithm which stabilizes the temperature of heated water, then by setting the flow rate of the delivered combustion air on such values corresponding the range of optimal air excess we can guarantee optimal operating conditions characterized by the minimal fuel consumption and by a compromise in lowest harmful emissions. This is the task that has been investigated on experimental setups.

\section{EXPERIMENTAL EQUIPMENT}

Most of experiments was performed on the commercially available $25 \mathrm{~kW}$ boiler. The boiler is designed for combustion of wooden and alternative (e.g. grain, hay) pellets of diameter 6 $-8 \mathrm{~mm}$. Nominal power output of the boiler is $25 \mathrm{~kW}$ for wooden pellets and $18 \mathrm{~kW}$ for alternative pellets. For all experiments have been used $6 \mathrm{~mm}$ softwood pellets. The boiler consists of lined combustion chamber located in the bottom section and system of heat exchangers in the upper section. In the combustion chamber is placed steel grate with primary air inlet from beneath and secondary air inlet holes located in the side walls of the chamber. The primary/secondary air ratio is adjustable by a flap. The pellets are fed from storage container by screw feeder to the rear side of the grate where they start to burn. Using the original control unit of the boiler, feeding of the pellets takes place periodically with preset periods of screw movement and idle state. During the operation, also the grate is periodically moved (swept) in the way that the pellets are moved towards front side of the combustion chamber. In optimal case, the pellets are completely burned out when they reach the end of the chamber. The ash is afterwards collected in the ash container. Both time intervals of fuel feeding and grate movement can be manually adjusted. The combustion air is fed into the boiler by air fan. The air fan is originally impulsecontrolled and allows manual adjustment of four different air flow rates. The boiler has been equipped with several measurement and indication points, as also shown in Fig. 2. In the original setting, none of these additional sensors is available. There are originally only two sensors detecting temperature of outlet water and flue gas. Responses from these sensors are used in the pre-programmed electronics for simple control purposes. The experimental setup has involved measurement of temperatures at the front of the combustion chamber (T1), temperature after first convective section (T2), flue gas temperature (T3), temperature of inlet (T4) and outlet water (T5) and oxygen and $\mathrm{CO}$ concentrations in the flue gas and flow rate of the combustion air. Additionally, there have been detected periods of grate sweeping and fuel feeding. Scheme of the boiler with the instrumentation is shown in Fig. 2.

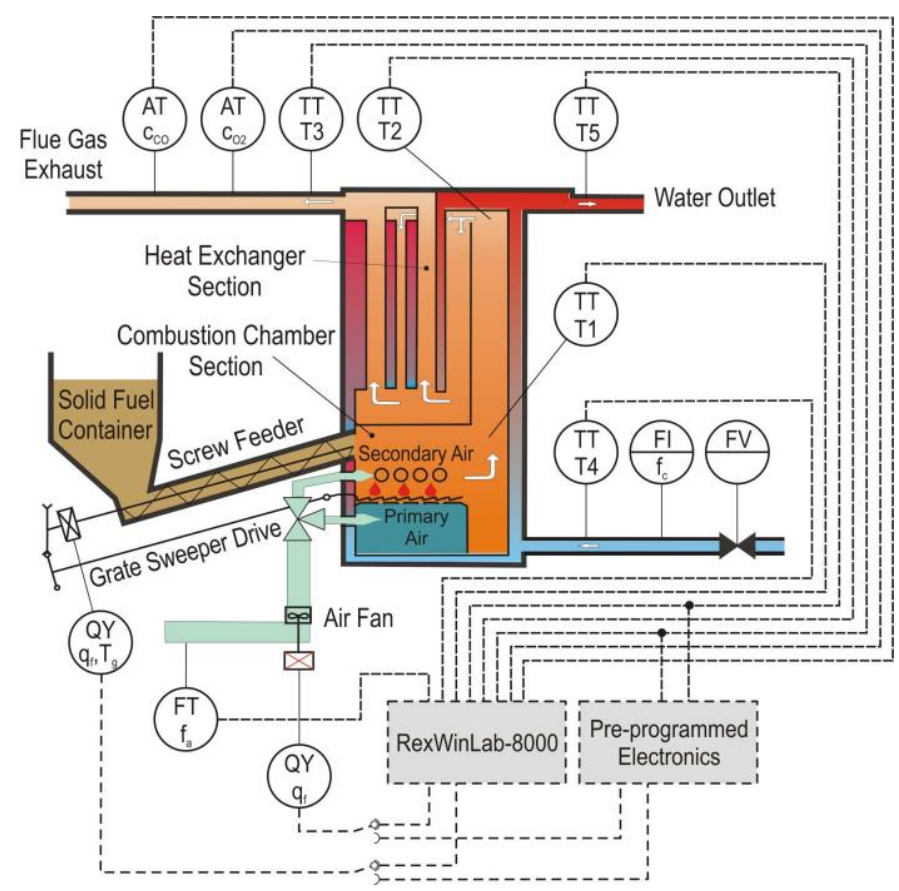

Fig. 2. Scheme of the experimental boiler with additional instrumentation ([2], fig 1)

The boiler was originally equipped with manufacturer's control unit that allowed only very limited intervention into the process. However, the original control unit still needs to be used, mainly for educational purposes. Therefore a new switchboard was designed and produced. The switchboard contains circuits assuring protection against overload, shortcircuit and forbidden combination of inputs, power sourcing, noise shielding, central earth and emergency stop function. The switchboard is also equipped with RexWinLab-8000 control and data acquisition unit. The control of combustion process can be assured either with pre-programmed electronics or switched to RexWinLab 8000. 

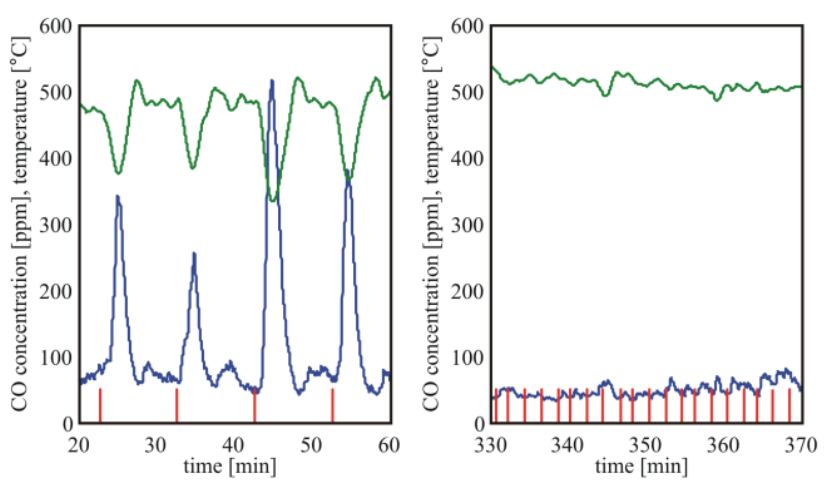

- concentration of $\mathrm{CO}$ in flue gases [ppm], — grate sweeping time instant [binary] temperature of flue gases beyond first heat exchanger $\left[{ }^{\circ} \mathrm{C}\right]$

Fig. 3. CO concentration peaks elimination by means of a better algorithm of the grate movement ([2], fig 2)

The RexWinLab-8000 is a control and data acquisition unit developed along with the switchboard at Department of Instrumentation and Control Engineering at the Czech Technical University in Prague. The RexWinLab 8000 is based on PAC (Programmable Automation Controller) Wincon 8000 series, but the control firmware of PAC is replaced by software named REX Control developed at Institute of Cybernetics at University of West Bohemia in Pilsen. REX Control allows using advanced control algorithms commonly unavailable in standard controllers. It has also its own graphical user interface based on similar principles as Simulink produced by MathWorks. Another useful feature of the software is its ability to communicate with Matlab/Simulink software bundle in realtime and exchange the data with it.

The described configuration allowed preparation of experiments in advance on a standard personal computer. Any control algorithm synthesis can be easily realized in graphical development environment very similar to well known Simulink. Data from all sensors placed in the boiler sensors are time-synchronized and centralized to PAC. During the experiment PAC sends measured data to Simulink running on remote computer and show courses of measured quantities in real-time. This method allows operator to monitor and potentially interfere combustion process during the experiment. Data are periodically saved in Simulink for the follow-up offline analysis. The described development of switchbox with RexWinLab 8000 changed standard factory boiler to an experimental base that allowed us to prepare, monitor and interfere, acquire data and analyze them during the experiments in real-time.

For these reasons the same concept has been used in a new prototype boiler "Fiedler $100 \mathrm{kW"}$. This boiler has been delivered recently and that is why we cannot present experimental results yet. Otherwise, the boiler is designed as a prototype using new technological principles and rich instrumentation capable to obtain necessary data for creating a simulation model. The simulation model is necessary for tuning control algorithms because the real-time testing of intended automated search for optimal operating conditions costs much time, money and effort

\section{MAIN EXPERIMENTALLY VERIFIED IMPROVEMENTS}

When we defined research project using a new prototype of $100 \mathrm{~kW}$ boiler we could come out from the following findings obtained and experimentally verified on the experimental Verner boiler:

\section{A. Grate Movement}

In small scale boilers the grate sweeping movement influences combustion process in dependence on in the way how it is carried out. In the described pilot boiler if fixed preprogrammed control units were used disturbing periodical strong peaks in the combustion process occurred during operation for a short time after the grate sweeping. The peaks depicted in Fig. 2 on the left could be removed (as shown in the right part of the figure) if another timing of this movement has been programmed and generated by a programmable controller. [3]

\section{B. Replacement of On-Off control by PI controller}

In the most of small-scale pellet boilers for temperature control of heated water, switching in fuel supply rates is used. Turning off fans ensuring air flow through the boiler can stop boiler heating function for quite a long time without any problems with re-burning. Our experiments showed that use of a standard PI control may reduce fuel consumption, but the boiler must be equipped with necessary electronics for carrying out this control algorithm. [3]

\section{Optimization of operating conditions}

All boilers are characterized by the fact that heat transfer from the boiler to the heated medium depends on the temperature of the heated medium. Usually, this temperature is controlled. If it is achieved such steady state when the temperature of the heated medium is at its desired value while in the fuel supply it has been reached its minimum, than this state indicates an efficiency maximum. Very important is the fact that combustion process runs under optimal conditions not only from the economical viewpoint (fuel consumption) but as well from the viewpoint of ecological aspects because simultaneously $\mathrm{CO}$ emissions are in the case at the lowest level (see Fig. 3). [4]

The most promising ecological effect in the further development showed the direction $\mathrm{C}$ - operational optimization based on the extremum seeking approach. That is why we decided to investigate its properties and alternatives before implementing designed algorithm in the control system of the new prototype boiler.

\section{ARRANGMENT OF CiRCUITS FOR HEATING TEMPARATURE CONTROL IN PROTOTYPE BOILER"}

Control solutions of operating condition optimization have some common features. Always, there is a standard control circuit whose control variable - temperature of the heating water measured at the heat exchanger outlet in the case of the investigated prototype boiler (Fig. 4) - corresponds the desired value in steady states. It is possible to keep one the same desired value of the control variable while the values of the 


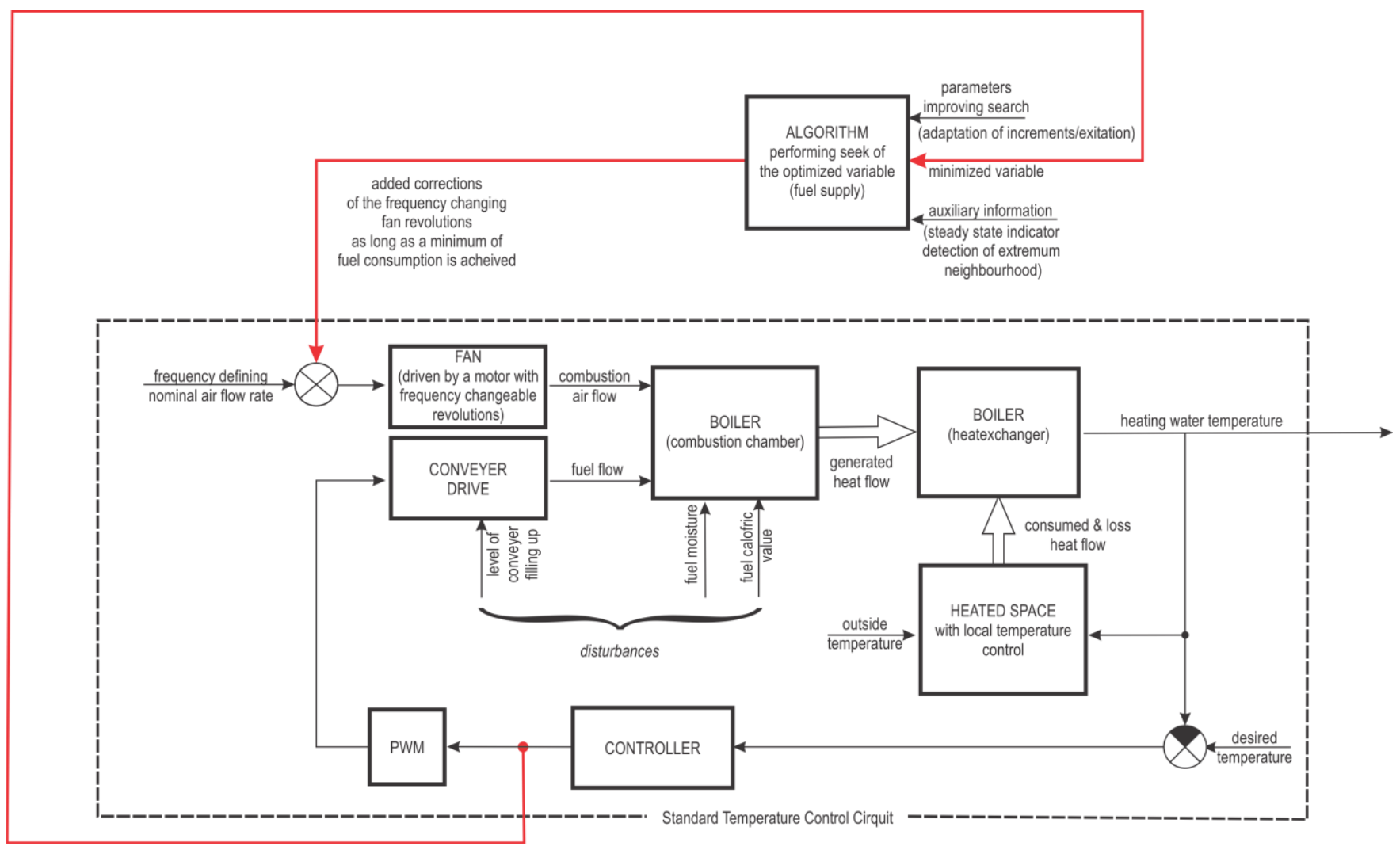

Fig. 4. Functional scheme of main components in the temperature control of the prototype biomass boiler equipped with the automated search of optimal operating conditions

manipulated variable mutually differ in dependence on another changeable variable influencing the efficiency of the whole controlled process.. In the case of combustion processes such variable is mostly the combustion air flow defining air surplus. In the boiler prototype scheme in Fig. 4 the air flow rate is not measured, it can be only changed by means of a frequency changer which makes possible to change revolutions of the fan drive. The extreme, in the case of efficiency it is a maximum, of course, is achieved when the fuel flow rate is at its minimum that can be detected as a lowest steady state value of the manipulated variable sufficient for keeping the control variable on the desired value. This value varies according to changing operating conditions usually having (dynamical) disturbance influence on the function of the (main) control circuit. [4]

A traditionally preferred main task of a system control design is to find a control algorithm and to set its parameters which guarantee the best responses of the controlled variable. Less attention is paid to the operating conditions. under which the controlled device works. These conditions strongly influence an overall efficiency of the controlled device and very often they determine the environmental impacts of controlled process, as it is in the case of a biomass combustion. Optimization of biomass-fired boiler operating conditions is therefore appropriate not only in order to increase the economic efficiency of the controlled system run, but also to fulfill tightening emission standards.

Suppose that the main system control is carried out by some main controller. The operating conditions optimization may be applied as a supplement to the traditional system control provided that, firstly, it is possible to manipulate with least one of the controlled system inputs different from the main controller control variable, and secondly, there are some identifiable optimal operating conditions. The first condition implies that the controlled system is a multi-input process. The second condition assumes that we can determine so-called cost function defining the operating conditions optimality based on defined criteria.

The biomass combustion in a hot-water boiler is a complex non-linear process with two main inputs - the fuel supply and the combustion air supply. When the fuel supply is widely used for a boiler output modulation, i.e. the primary boiler control, then the combustion air supply may be used for the operating conditions control. Control to a constant air-fuel equivalence ratio $(\lambda)$ is usually used in large and medium-scale boilers for the operating conditions control by the control of the combustion air supply. The control to the constant $\lambda$ requires a special wide-band lambda sensor whose increasing availability allows the use of this kind of control even for small-scaled boilers. The optimization of the operation conditions controlled by the constant $\lambda$ control lies in the measuring of the optimal value of $\lambda$ for a specific device in advance, i.e. so called offline optimization.[5]

The main disadvantage of the off-line optimization is the necessity to know the optimal value of the monitored parameter in advance. In biomass combustion the optimal value of $\lambda$ is not constant. It depends on many variable 
parameters, mostly parameters of the fuel. Moreover, these fuel parameters, such as fuel moisture, or fuel caloric value to name those depicted as examples in Fig. 4, are practically immeasurable on-line, and thus any pre-set of the optimal values of $\lambda$ for different states of the combustion process is unfeasible. A real-time operation conditions optimization does not suffer from this disadvantage. However, the real-time optimization is based on an on-line process monitoring and an evaluation of the current optimal value of the controlled secondary input from on-line measured data. The real-time optimization therefore represents much more complex problem then the off-line optimization. [6]

\section{REAL-TIME OPTIMIZATION BY EXTREMUM-SEEKING CONTROL}

An extremum seeking control or a self-optimizing control is set of control problems dealing with tracking a varying extremum (maximum or minimum) of a cost (performance, objective, output) function. It is common that a reliable mathematical model of non-linear controlled plant is not available to predict the variation of the cost function with time. A strength of the extremum seeking control is that an only necessary knowledge is the cost function attains an extremum (maximum or minimum) and this extremum is reachable by setting of an input $u$. Any explicit knowledge about the plant or its steady-state input-output map is not necessary. The extremum seeking control is therefore a model-free on-line optimization gradient-based method that dynamically seeks the optimal point of input-output steady state mapping of the plant. [6]

\section{A. Continue Extremum-seeking Control Methods}

The continue approach utilize the perturbation signal added to the controlled plant input to excite the controlled plant and explore the controlled plant steady-state map. The current gradient of the cost function is then evaluate from a continue observation of the control plant output. The controlled plant input is then continuously change on the basis of the evaluated cost function gradient $[7,8]$.

\section{B. Numerical Extremum-seeking Control Methods}

Iterative extremum-seeking methods use a discrete sequence of probing inputs and then utilize methods of numerical optimization to reach the cost function extremum. In contrast to the continue methods the numerical extremum-seeking algorithms do not require the continues observation of the control output and the optimization algorithm may have time to collect enough information to the cost function gradient estimation [8].

\section{Simulation MOdel OF BiOMASS-FIRE BOILER FOR TESTING OF MiniMUM-SEEKING ALGORITHMS}

A functionality of the proposed biomass-fired boiler operation optimization based on minimum-seeking algorithm was proved on small-scale biomass-fired boiler as mentioned above. The minimal iterative algorithm based on relay principal was used during functionality proving experiments.
A set of promising minimum-seeking algorithms, which can be used for the biomass-fired boiler operation optimization, includes a large variety of different algorithms. Moreover, each algorithm includes more or less parameters that need to be pretuned for a specific application. It would be very timeconsuming to test and tune all possible algorithms or develop new algorithms on a real-world biomass-fired boiler. This resulted in the need to carry out first investigation in a simulated way. A model for this purposes has been proposed with the assumption that it is not necessary (and possible) to design a precise physical-mathematical model of biomass combustion process and heat transfer in a biomass-fired hotwater boiler. For testing and designing proposes it is enough if the optimization algorithms are developed on the basis of a simulation model which represents the real object in an approximate way.

Behavior of such simulation model must include an estimate of the static sensitivity, essential dynamics and nonlinear character of responses at least for the input used in optimization.

The block scheme of the proposed simulation model is depicted in Fig. 5. Combustion and heat transfer processes are modeled by means of two transfer functions reflecting empiric knowledge of the real boiler dynamics. By these transfer functions, the modeled dynamics is caught in differences of variables from their steady state absolute values which in the model are added to the differences as outputs from the blocks with dotted borders. These blocks serve as sources of constant values readable inside of such blocks. The transfer blocks in the lower part of the complex block bearing the label BOILER (the controlled system) have three inputs: the fuel feeding flow obtained as converted values of the manipulated variable $u$ generated by the PI controller; then the efficiency impact of the current operating conditions generated as an dynamically delayed output from a block generating current values of efficiency defined as a nonlinear tabulated function; and the third input represent noise and the other disturbances that are not depicted in the scheme. As the output of the whole block BOILER considered as the controlled variable $y$ is the heating water temperature. Values of the controlled variable $y$ are input for block representing the PI controller block where they are compared with the desired value of the heating water temperature differences between. The PI controller evaluates the control error $e$ and according to its algorithm sets the values of fuel feeding necessary to reach and keep the heating water

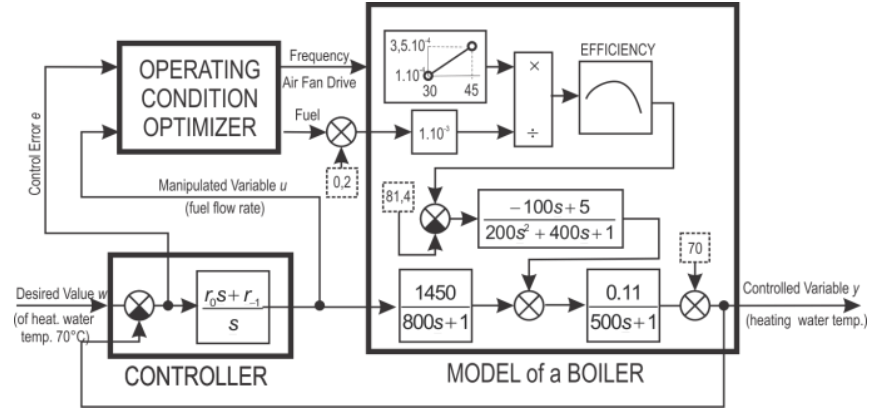

Fig. 5. Block scheme of simulation model approximation roughly reflecting a biomass-fired boiler behavior used for testing and evaluating different minimum-seeking algorithms 
temperature $y$ on the desired value $w$. The looking-up of efficiency values is based on evaluating the ratio air-fuel. The air flow rate changes are derived from the frequency of the electric current supplying the air fan drive. The frequency is calculated in the block OPERATING CONDITIONS OPTIMIZER. In the ratio calculation it is important not only knowledge of the current value of the fuel delivery but also to detect to the controller steady states as suitable instants for performing frequency changes. Therefore the control error $e$ is the next input for the optimizer making possible to speed up the steady state recognition.

Thus designed simulation model was realized in Matlab Simulink. Parameters of blocks used in the simulation program are based on experiments and empiric experience obtained in laboratory instillation of small-size boilers. The looked-up combustion efficiency value undergoes a dynamic transfer which is done by a non-minimal phase block of the second order. This reflects an observed behavior when a response of the output water temperature to the step change in air intake value starts in the opposite direction before changes its course towards to a new steady state value.

\section{MinIMUM-SEEKING ALgORITHM APPLICABILITY TESTS}

The extremum-seeking based optimization methods are divided to two groups, the continue methods and the iterative methods, as mentioned before. Our goal now is to evaluate advantages and shortages we can expect implementing algorithms from the first or second group. We carried out a set of simulation experiments using the simulation model of a biomass-fired boiler, which was described in the previous section VI. These experiments had to assess applicability of both main optimization approaches. In these tests we intended to speed, accuracy, precision and robustness comparison. Schemes representing both main extremum-seeking based optimization approaches are depicted in Fig. 6 and Fig. 7 depicted.

The scheme in Fig. 6 represents the group of numerical-based extremum-seeking methods. The gradient estimator evaluates the impact of an increment of the fan drive frequency on the change of the fuel feed. Both differences are evaluated after achieving the new steady-state. The steady-state is evaluated from trends of the control error. A numerical optimization algorithm then computes the new fan drive

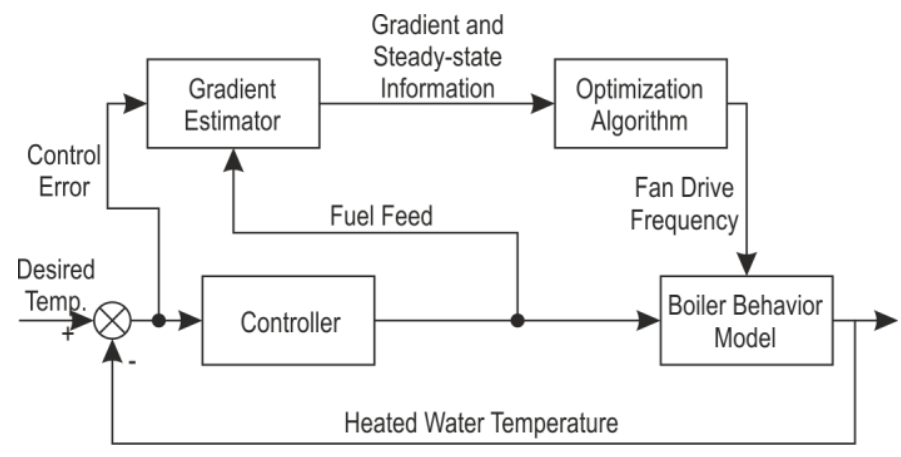

Fig. 6. Scheme of the optimizer using a numerical-based extremum-seeking method frequency by the steepest descent formula:

$$
u_{f a n}(k+1)=u_{f a n}(k)+\varepsilon \frac{d u_{f u e l}}{d u_{f a n}(k)}
$$

where $u_{f a n}(k+1)$ is the new value of the fan drive frequency, $u_{f a n}(k)$ is the current value of the fan drive frequency, $\varepsilon$ is update constant and $d u_{f u e l} / d u_{f a n}(k)$ is the estimation of the gradient in the current step.

The scheme in Fig. 7 presents a continuous method representative. This algorithm is based on a general continues extremum-seeking scheme presented in [6]. It uses a harmonic sinusoidal perturbation excitation added to the electric current frequency driving the air fan motor. When the frequency of the harmonic perturbation is slow enough, then the dynamics of the heating water control loop does not interfere with the extremum-seeking loop and this control loop can be described as a static input-output map. Detail arrangement of the

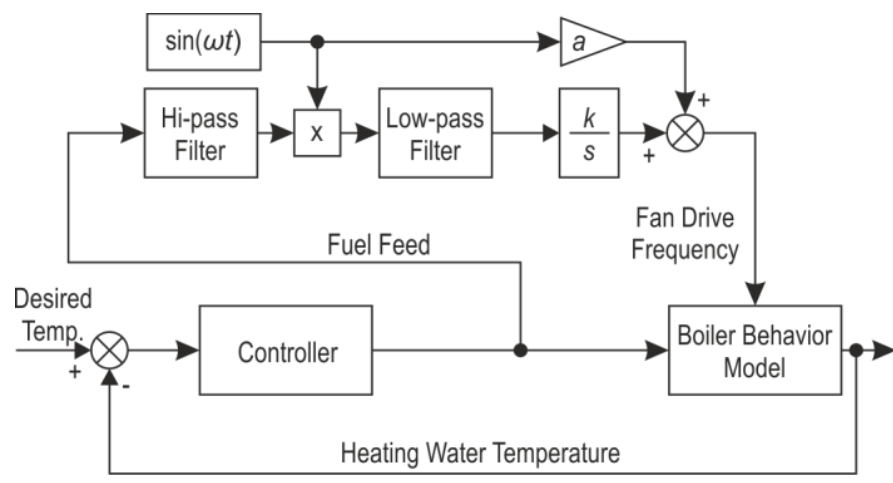

Fig. 7. Scheme of the optimizer using a continue extremum-seeking method

extremum-seeking scheme in Fig. 7 shows that the excitation signal $a \sin (\omega t)$ is fed to the Fan Drive input and it excites the sinusoidal response of the output Heating Water Temperature. This response after processing in the controller is filtered by a high-pass filter with transfer function

$$
F_{\text {high-pass }}=\frac{s}{s+\omega_{h}}
$$

Both the output of the high-pass filter and the excitation signal are nearly two sinusoids that are in phase or out of phase. If the static input-output map is convex (the extremum is minimum), then "out of phase" means that a current Fan Drive Frequency is lower than the target optimal frequency. If it is "in phase" it means that a current Fan Drive Frequency is higher than the target optimal frequency. If the shape static input-output map is concave, the reverse holds true. The product of both sinusoids contains a "DC component". This "DC component" is extracted by the low-pass filter

$$
F_{\text {low-pass }}=\frac{\omega_{l}}{s+\omega_{l}}
$$



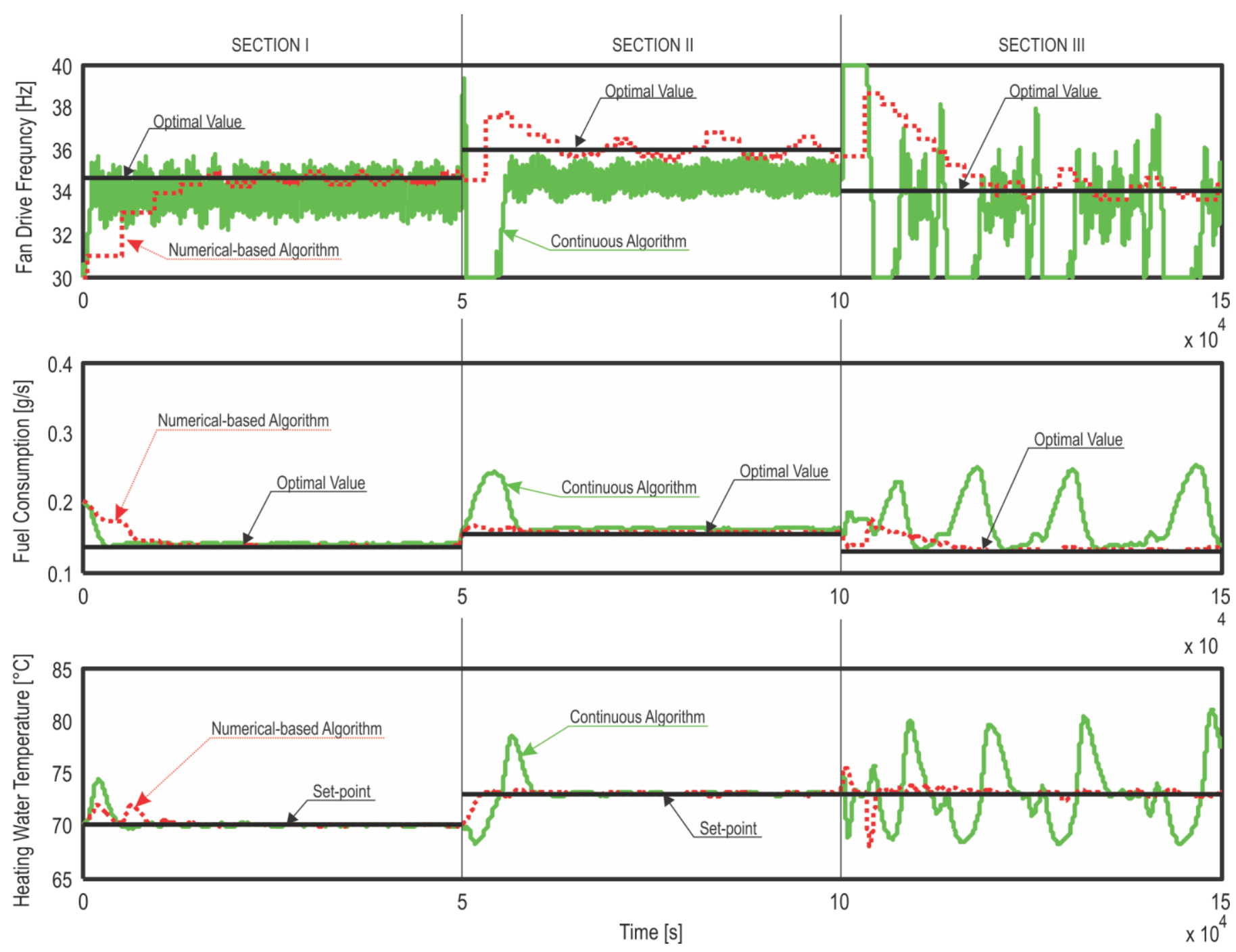

Fig. 8. Simulation experiment results comparing two different extremum-seeking optimization methods show

- in Section I, a good feasibility of both methods to reach desired an optimal steady-state when they were started from non-optimal operating conditions;

- in Section II, better responses to a change (from $70^{\circ} \mathrm{C}$ to $73^{\circ} \mathrm{C}$ ) of the heating water temperature desired value in the case of the continues algorithm;

- in Section III, an influence of changes in the air supply on the boiler behaviour if the air supply conditions have changed

The integrator is then update law tunes current input value to target input value driven by the sensitivity function. A general form of the optimization algorithm formula is:

$$
\begin{aligned}
& u_{f a n}(t)=u_{f a n}(0)+a \sin (\omega t)+ \\
& +u_{f u e l}(t) \frac{s}{s+\omega_{h}} \sin (\omega t) \frac{\omega_{l}}{s+\omega_{l}} \frac{k}{s}
\end{aligned}
$$

The simulation experiments were aimed mainly to assess the precision, the accuracy and the robustness of both methods for optimization of described system. A representative result of tests is in Fig. 8 depicted. In this figure are three graphs. A permitted range of the fan drive frequency values was limited to the range from 30 to $40 \mathrm{~Hz}$.

The first graph in Fig. 8 shows the course of the electric current frequency driving the air fan motor which is the output of the optimization algorithms and on of the inputs of the boiler behavior model. The course of the fuel feed needed to maintain the desired heating water temperature is captured in the middle graph. The bottom graph shows the course of the heating water temperature that is to be maintained at the desired value. The solid line belongs to the continuous optimization method and the dotted line belongs to the numerical-based method. The black solid lines shows the optimal values of variables in the first and second graph. The desired temperature of heating water is plotted using a solid type of the line labeled as "Setpoint'.

All three graphs are divided to three sections. The first section shows the start of optimization. The starting conditions of the boiler behavior model are different from those representing desired optimal operating state. In the beginning of the simulation experiment, the fan drive frequency is set to the lowest permissible limit value of $30 \mathrm{~Hz}$ and the heating water control circuit is in steady state. This section of experiment was aimed to show the speed, accuracy and precision of both methods. It confirmed that the used 
continuous method was much faster than the used numerical-based method. Whereas the numerical-based method needs to wait until the heating water control loop realizes a steady state after each step, the used continuous method utilizes continuous gradient estimation and reaches the optimal value in time which is close to the control loop dynamics. On the other hand, the numerical-based method can be assessed as both precise and accurate. The continuous method based on the sinusoidal excitation cannot be accurate and precise at once. It is needed to find a compromise between precision and accuracy during the used continuous optimizing algorithm tuning because the simulation experiments showed that the relationship between the accuracy and the precision is inversely proportional with this method.

The desired heating water temperature was changed from $70{ }^{\circ} \mathrm{C}$ to $73{ }^{\circ} \mathrm{C}$ in the beginning of Section two. Immediately after this change, the controller starts to change of the fuel feed in order to reach the new desired value of the heating water temperature. The need of the numerical-based method to wait for a steady-state is an advantage in this situation. The continuous method reacts to changes in fuel consumption forced by the change of the desired heating water temperature as this changes are the reaction of the control loop to the optimizing algorithm excitations. Only the limitation of the fan drive frequencies in the range from 30 to $40 \mathrm{~Hz}$ kept the system stable. Moreover, because the change of the control loop operation point changes its dynamics and sensitivity, the used continuous method is less accurate (but more precise). The accuracy of the numerical-based method remained the same.

The third section characterizes change in the air supply conditions carried at its begin. They changed the behavior of the boiler so much the used continuous optimization method collapsed and needs to retune the parameters for new operation conditions. The numerical-based method is slightly imprecise but still able to achieve and safely maintain the new optimal value of the fan drive frequency under the new operating conditions.

\section{CONCLUSIONS}

The aim of the paper is to show some of the issues solved as ecological improvements suitable for small-scale boilers. Considered solutions had to be in the range of low-cost, because no expensive instrumentation is acceptable with respect to the total price of the boiler. Therefore we focused at design of algorithms for PLC's. Their transfer and modification are tested when applied for a medium size prototype boiler we have newly at disposal for experiments in our labs.

From all the algorithms tested on the small-scale boiler, the most suitable for transfer are the algorithms optimizing combustion. Due to a different construction of both boilers it is necessary to define steps of the transfer based on the partial experiments.

One of such transfer steps is investigation of algorithms performing search of optimal operating conditions. This has been done with use of a simplified model of boilers. Control of one or more variables, that is linked with the requirement to keep optimal operating condition by sliding manipulation of another manipulated variable, is a task which can be found in the other devices. Therefore its reliable solution has enough opportunities to be applied in other control tasks.

The simulation experiments show that the continuous-based algorithms are faster then numerical-based algorithms but are much more sensitive to disturbances and to changes in the control loop parameters. Because both, disturbances and control loop parameters parameter changes, are often occur in the process of biomass combustion the numerical-based approach seems to be more suitable for the proposed optimization.

Further experiments and algorithm design will be aimed especially at improving numerical-based optimization approach including the current operation state estimation and its future state prediction.

\section{ACKNOWLEDGMENT}

This research had support within Project MSM 68400 770035 "Development of environmentally friendly decentralized power systems".

Latest results have achieved within the project TA02020836 "Inteligent control methods of residual-biomass fired boiler ecological control" having financial support from Technology Agency of the Czech Republic.

The work of Ph.D. students has been supported by the Doctoral Grant Support of the Czech Technical University in Prague, grant No. SGS13/179/OHK2/3T/12

\section{REFERENCES}

[1] J. Hrdlicka a B. Sulc, „On-line operating adjustment of small biomass fired boilers optimizing CO and NOx emissions", in Proceedings of the 6th IASME/WSEAS international conference on Energy \& environment, Stevens Point, Wisconsin, USA, 2011, p. 35-40.

[2] V. Placek and J. Hrdlicka, "Influence of control on environmental and economical aspects of small-scale biomass boiler," in 2011 International Conference on Power Engineering, Energy and Electrical Drives (POWERENG), 2011, pp. 1-3.

[3] Šulc, B., Vrána, S., Hrdlička, J., Lepold, M. (2009). Control for Ecological Improvement of Small Biomass Boilers, IFAC Symposium Power Plants \& Power Systems, 5-8 July, 2009, Tampere, Finland.

[4] Oswald, C., Šulc, B. (2011). Achieving Optimal Operating Conditions in PI Controlled Biomass-fired Boilers: Undemanding way for improvement of small-scale boiler effectiveness. In: Proceedings of the 2011 12th International Carpathian Control Conference. Velke Karlovice, 25.-28. May 2011. pp 280-285. ISBN: 978-1-61284-359-9

[5] Van Loo, S., Koppejan, J., editors (2008). The Handbook of Biomass Combustion and Co firing. London: Earthscan, 2008.

[6] C. Oswald,. V. Plaček, B. Šulc, A. Hošovský: Transfer Issues of Control Optimizing Combustion from Small-scale to Medium scale Biomass-fired Boilers. 8th IFAC Symposium on Power Plant and Power System Control. Toulouse 2012. ISBN: 978-3-902823-24-3. ISSN: 1474-6670

[7] Ariyur, K.B., Krstić, M.. (2003) Real-Time Optimization by ExtremumSeeking Control. John Wiley \& Sons, Inc., Hoboken, New Jersey, 2003. ISBN 0-471-46859-2

[8] Zhang, C., Ordóñez R. (2012), Extremum-Seeking Control and Applications: Applications: A Numerical Optimization-Based Approach. Advances in Industrial Control, Springer London, 2012. ISBN 978-14471-2223-4

Creative Commons Attribution License 4.0 (Attribution 4.0 International, CC BY 4.0)

This article is published under the terms of the Creative Commons Attribution License 4.0

https://creativecommons.org/licenses/by/4.0/deed.en_US 